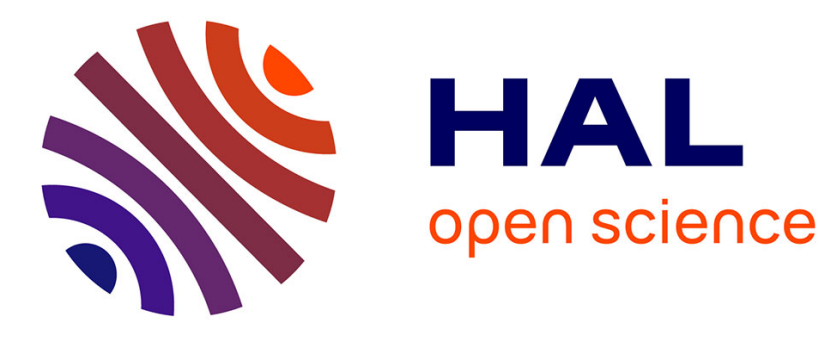

\title{
A comparison of iterative receivers for the non linear satellite channel
}

\author{
Bouchra Benammar, Nathalie Thomas, Charly Poulliat, Marie-Laure \\ Boucheret, Mathieu Dervin
}

\section{- To cite this version:}

Bouchra Benammar, Nathalie Thomas, Charly Poulliat, Marie-Laure Boucheret, Mathieu Dervin. A comparison of iterative receivers for the non linear satellite channel. 16th Workshop on Signal Processing Advances in Wireless Communications (SPAWC 2015), Jun 2015, Stockholm, Sweden. pp. 520-524. hal-01461702

\section{HAL Id: hal-01461702 https://hal.science/hal-01461702}

Submitted on 8 Feb 2017

HAL is a multi-disciplinary open access archive for the deposit and dissemination of scientific research documents, whether they are published or not. The documents may come from teaching and research institutions in France or abroad, or from public or private research centers.
L'archive ouverte pluridisciplinaire HAL, est destinée au dépôt et à la diffusion de documents scientifiques de niveau recherche, publiés ou non, émanant des établissements d'enseignement et de recherche français ou étrangers, des laboratoires publics ou privés. 


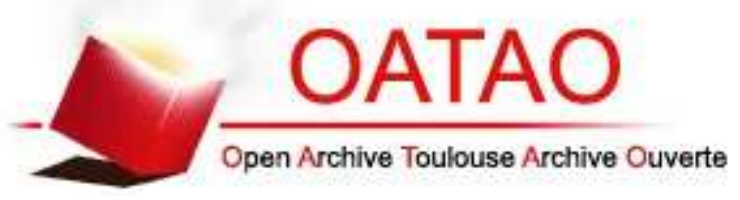

\section{Open Archive TOULOUSE Archive Ouverte (OATAO)}

OATAO is an open access repository that collects the work of Toulouse researchers and makes it freely available over the web where possible.

This is an author-deposited version published in : http://oatao.univ-toulouse.fr/ Eprints ID : 17192

The contribution was presented at $\mathrm{XX}$ :

http://www.spawc2015.org/

To cite this version : Benammar, Bouchra and Thomas, Nathalie and Poulliat, Charly and Boucheret, Marie-Laure and Dervin, Mathieu $A$ comparison of iterative receivers for the non linear satellite channel. (2015) In: 16th Workshop on Signal Processing Advances in Wireless Communications (SPAWC 2015), 29 June 2015 - 1 July 2015 (Stockholm, Sweden).

Any correspondence concerning this service should be sent to the repository administrator: staff-oatao@listes-diff.inp-toulouse.fr 


\title{
A comparison of iterative receivers for the non linear satellite channel
}

\author{
Bouchra Benammar*, Nathalie Thomas ${ }^{\dagger}$, Charly Poulliat ${ }^{\dagger}$, Marie-Laure Boucheret ${ }^{\dagger}$, Mathieu Dervin ${ }^{\ddagger}$ \\ *Technological Research Institute (Saint-Exupery), Toulouse France \\ bouchra.benammar@irt-saintexupery.com \\ †University of Toulouse, INPT-ENSEEIHT/IRIT, Toulouse France \\ \{nathalie.thomas, charly.poulliat, marie-laure.boucheret\}@enseeiht.fr \\ $\ddagger$ Thales Alenia Space, Toulouse, France \\ mathieu.dervin@thalesaleniaspace.com
}

\begin{abstract}
This paper presents a comparison of different instances of advanced iterative receivers for the non linear satellite channel. A comparison of the performance and complexity of each of the selected receivers is drawn. It is shown that the frequency domain implementation of the linear equalizer achieves good performance complexity trade-off. The cost to pay for the frequency domain processing is the addition of a Cyclic Prefix (CP) to ensure the blocks orthogonality. The consequence is a channel dependent spectral efficiency loss. We thus investigate on the efficiency gain related to the $\mathrm{CP}$ omission for frequency domain equalizers for different block sizes and show that for large block sizes, the equalizer's performance is not much sacrificed.
\end{abstract}

\section{INTRODUCTION}

New generation satellite services are required to provide higher throughputs to cope with the increasing demand for data rates. In band-limited systems, the increase of spectral efficiency is the key to improve the transmission throughput. In this context, the Extension of the Digital Video Broadcasting - Second generation (DVB-S2X) standard [1] suggests using very low roll-off shaping filters in conjunction with new modulation schemes up to hundreds of symbols per constellation. If in theory, a gain up to $15 \%$ can be achieved in spectral efficiency, the real achievable gains are less optimistic due to satellite channel impairments. Indeed, using high modulation orders such as Amplitude and Phase Shift Keying (APSK) and low roll-offs leads to higher signal fluctuations. Thus, amplifiers on board satellite transponders need to be operated far from their saturation region in order to limit the distortions caused by the clipping effect of the amplifier. However, the larger the Input Back-Off (IBO) towards saturation, the poorer the energy efficiency of the satellite transponder. In practice, the back-off allowing the best trade-off between energy efficiency and amplifiers distortions can be decreased using adequate processing of the non linear distortions.

Provided that the non linear distortions are suitably modelled, their mitigation can be carried out either at the transmitter (using the so-called pre-distortion techniques) or at the receiver (using equalization). As far as pre-distortion is concerned, the non linear interference can be applied to the signal or to data it carries which can change the statistical and spectral properties of the transmitted waveform so that it may sometimes no longer be compliant with the transmission mask. Thus we are interested in this article in receiver processing techniques and more specifically in iterative equalizers.

In [2], a symbol based non linear channel model is derived leading to the so-called non linear Inter Symbol Interference (ISI) Volterra model. This model has allowed the derivation of optimal symbol and sequence receivers [3], [4]. However, the complexity of the optimal equalizers scaling exponentially with the channel memory length, sub-optimal receivers have been investigated. In [5], a factor graph based equalizer is derived for a specific simplified structure of the linear satellite channel with complexity increasing only linearly with channel memory. As far as linear equalizers are concerned, we have derived in [6], [7] linear time and frequency domain iterative equalizers for general Volterra channels pointing out the complexity challenges inherent to the complexity of the Volterra model itself.

The motivation behind this paper lies in the lack of a general comparison between the numerous instances of iterative receivers proposed for the non linear channel. Thus, we propose to investigate the performance of optimal and suboptimal equalizers and some of their reduced complexity implementations, and analyse the influence of system parameters such as the filters length and the cyclic prefix size on the receiver behaviour. The remainder of this article is organised as follows: In Section II, we present the symbol based Volterra model for the non linear satellite channel. This model allows for the expression of different equalizers in Section III. Section IV presents a comparison of the different realisations of the iterative equalizers before ending up with some conclusions. Useful notations: In the sequel, vectors and matrices are represented by lower-case and upper-case bold letters respectively. The notation $\mathbf{0}_{a \times b}$ designates the size $a \times b$ all zero vector. $\mathbb{I}_{N}$ designates the $N \times N$ identity matrix.

\section{NON LINEAR SATELlite CHANNEL}

Transmission using satellite services is subject to linear and non linear distortions owing to the processing on-board the satellite transponder. To model these distortions, let us consider the base-band transmission scheme depicted in Fig. 1. A stream of independent identically distributed bits is passed though a channel coder, interleaved and forwarded to a mapper 


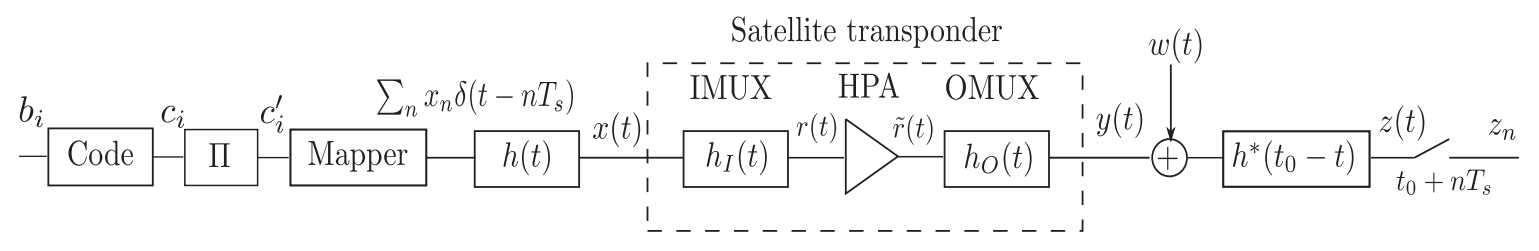

Fig. 1. Base-band satellite transmission chain

which merges blocks of $\log _{2}(\mathcal{M})$ bits into complex symbols $x_{n}$. These symbols are then up-sampled to a symbol period $T_{s}$ and linearly filtered by a shaping filter $h(t)$ yielding the transmit signal $x(t)$. This signal is sent to a satellite transponder which amplifies and redirects the uplink sub-band signal to the corresponding downlink channel and is thus composed of three processing stages. The Input MultipleXer (IMUX) is a band-pass filter which filters out the undesired adjacent channels, the power amplifier amplifies the input signal to a desired output power following the requirements of the link budget, and finally an Output MultipleXer (OMUX) mitigates out-of-band spectral regrowth in order not to interfere on adjacent channels at the downlink. The uplink noise and adjacent channels interferences are neglected. The satellite power amplifier is typically a Travelling Wave-Tube Amplifier the response of which is characterised by two memoryless functions relating the amplitude of the input signal to both the amplitude (AM/AM) and phase (AM/PM) of the output signal. The complex envelope of the signal at the output of the amplifier writes as follows:

$$
\tilde{r}(t)=A(|r(t)|) \exp ^{j(\phi(t)+\Phi(|r(t)|))}
$$

where the functions $A($.$) and \Phi($.$) describe the AM/AM and$ $\mathrm{AM} / \mathrm{PM}$ responses respectively and $\phi(t)=\arg (r(t))$. Although the amplifier response is memoryless, its combination with on-board and transmission filters, induces some memory in the satellite channel. In [2], a symbol-based non linear channel with memory has been derived for bandpass non linear satellite channels using an odd-order Volterra series decomposition which yields received symbols writing as follows:

$$
\begin{aligned}
z_{n} & \triangleq z\left(t_{0}+n T_{s}\right) \\
= & \sum_{i=0}^{v} \gamma_{2 i+1} \sum_{n_{1}} \ldots \sum_{n_{i+1}} \sum_{n_{i+2}} \ldots \sum_{n_{2 i+1}} x_{n-n_{1}} \ldots \\
& x_{n-n_{i+1}} x_{n-n_{i+2}}^{*} \ldots x_{n-n_{2 i+1}}^{*} K\left(n_{1}, \ldots, n_{2 i+1}\right)+w_{n}
\end{aligned}
$$

where $v$ specifies the decomposition order of the Volterra series, $\gamma_{i}$ are decomposition factors related to the power amplifier response, $w_{n}$ a sampled white noise with variance $\sigma_{w}^{2}$, and $K\left(n_{1}, \ldots, n_{2 i+1}\right)$ are called Volterra kernels and can be expressed as:

$$
\begin{gathered}
K\left(n_{1}, \ldots, n_{2 i+1}\right)=\int \tilde{h}_{I}\left(t+n_{1} T s\right) \ldots \tilde{h}_{I}\left(t+n_{i+1} T_{s}\right) \\
\tilde{h}_{I}^{*}\left(t+n_{i+2} T_{s}\right) \ldots \tilde{h}_{I}^{*}\left(t+n_{2 i+1} T_{s}\right) \tilde{h}_{O}\left(t_{0}-t\right) d t
\end{gathered}
$$

where $\tilde{h}_{I}$ (resp. $\tilde{h}_{O}$ ) are the convolution of the transmit filter and IMUX (resp. receiver filter and OMUX). The obtained
Volterra kernels are then compressed, i.e. kernels leading to the same symbol combinations can be merged. For example, kernels with indexes $(i, j, k)$ and $(j, i, k)$ both lead to the same symbol product $x_{i} x_{j} x_{k}^{*}$, which can thus be merged as a unique kernel $h_{i, j, k}=K_{i, j, k}+K_{j, i, k}$. The Volterra model can be further simplified by taking advantage of the modulation symbols symmetries. More specifically, when the symbols satisfy $\left|x_{n}\right|^{2}=1$ typically for a PSK modulation, the Volterra kernels can be reduced by noting that products of symbols $(i, j, i)$ or $(i, j, j)$ contribute to first order kernels $j$ or $i$ respectively. The same reasoning can be applied to higher order kernels. Henceforth, the kernel indexes $(i, j, k)$ satisfy $(i, j) \neq k$ and $h(i, j, k)$ are given in a triangular form, i.e. $h_{i, j, k}=0$ if $j>i$. Furthermore, we shall restrict our analysis to causal third order Volterra decompositions with a symbol memory not exceeding $M$ which yields the following expression of the received symbols:

$$
z_{n}=\sum_{i=0}^{M-1} h_{i} x_{n-i}+\sum_{\substack{i \\(i, j, k) \in \mathcal{I}_{3}}} \sum_{\substack{k \\ j}} h_{i, j, k} x_{n-i} x_{n-j} x_{n-k}^{*}+w_{n}
$$

where $\mathcal{I}_{3}$ is a subset of the set $\{0, \ldots, M-1\}^{3}$.

\section{ITERATIVE RECEIVERS FOR THE VOLTERRA CHANNEL MODEL}

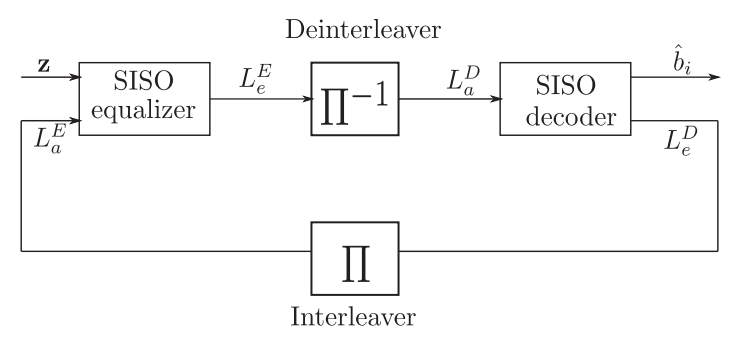

Fig. 2. Structure of a turbo equalizer

In this section we are interested in iterative receivers for the afore-presented Volterra model. A turbo equalization scheme as proposed in [8] and depicted in Fig 2 consists of two Soft Input Soft Output (SISO) processing blocks. A SISO equalizer computes Log Likelihood Ratios (LLR) of coded bits $c_{n, i}$ based on the channel observations $\mathbf{z}$ and on the input LLRs $L_{a}^{E}$ and a SISO decoder computes LLRs on coded and information bits. A SISO equalizer can be generally decomposed into two blocks:

- An equalizer which computes estimated symbol probabilities $P\left(x_{n}=\tilde{x} \mid \mathbf{z}, L_{a}^{E}\right)$. 
- A demapper which computes corresponding coded bits LLRs as follows:

$$
L_{a p p}^{E}\left(c_{n, i}\right)=\log \left(\frac{\sum_{\tilde{x} \in \mathcal{X}_{0}^{i}} P\left(x_{n}=\tilde{x}\left[\mathbf{z}, L_{a}^{E}\right)\right.}{\sum_{\tilde{x} \in \mathcal{X}_{1}^{i}} P\left(x_{n}=\tilde{x} \mid \mathbf{z}, L_{a}^{E}\right)}\right)
$$

where $\mathcal{X}_{j}^{i}$ corresponds to the set of symbols where the $i^{\text {th }}$ bit is equal to $j \in\{0,1\}$.

\section{A. Optimal detectors}

Optimal detection based on symbol representation of non linear ISI channels has been investigated both for symbol and sequence detection [3], [4]. To do so, the Volterra model has been represented by a finite state machine or equivalently by a trellis whose branch transitions outputs are non linear functions of the memory symbols. We shall restrain our analysis to symbol based Maximum A Posteriori detection and more specifically its efficient implementation using the Bahl-Cocke-Jelinek-Raviv (BCJR) algorithm [9].

\section{B. Linear Minimum Mean Square Error (MMSE) detectors}

In this section, we are investigating sub-optimal linear turbo equalizers for the non linear satellite channel. The vectorial representation of the Volterra channel model can be written as follows:

$$
\mathbf{z}_{n}=\mathbf{H} \mathbf{x}_{n}+\sum_{i} \sum_{j} \sum_{k} \mathbf{H}_{i j k} \mathbf{x}_{n}^{i j k}+\mathbf{w}_{n}
$$

where we define the following vectorial notations:

$$
\begin{aligned}
& \mathbf{z}_{n} \triangleq\left[z_{n-N_{1}}, \ldots, z_{n+N_{2}}\right]^{T} \mathbf{w}_{n} \triangleq\left[w_{n-N_{1}}, \ldots, w_{n+N_{2}}\right]^{T} \\
& \mathbf{x}_{n} \triangleq\left[x_{n-N_{1}-M+1}, \ldots, x_{n+N_{2}}\right]^{T}
\end{aligned}
$$

and where $\mathbf{H}$ is a linear convolution Toeplitz matrix having $\left[h_{M-1} \ldots h_{0}, 0 \ldots 0\right]$ as the first line, $\mathbf{H}_{i j k}=h_{i, j, k} \mathbf{I}_{N}$ are diagonal matrices and $x_{n}^{i j k}$ are the non linear interfering symbols writing as:

$$
\mathbf{x}_{n}^{i j k} \triangleq\left[\begin{array}{c}
x_{n-N_{1}-i} x_{n-N_{1}-j} x_{n-N_{1}-k}^{*} \\
\vdots \\
x_{n+N_{2}-i} x_{n+N_{2}-j} x_{n+N_{2}-k}^{*}
\end{array}\right]
$$

The MMSE equalizer as derived in [6] writes as a linear transformation of the received block symbols as follows:

$$
\hat{x}_{n}=\mathbf{a}_{n} \mathbf{z}_{n}+b_{n}
$$

where $\mathbf{a}_{n}$ and $b_{n}$ satisfy the MMSE criterion $E\left[\left|\hat{x}_{n}-x_{n}\right|^{2}\right]$. The MMSE estimated symbols write then as:

$$
\hat{x}_{n}=\mathbf{a}_{n}\left(\mathbf{z}_{n}-E\left[\mathbf{z}_{n}\right]\right)+E\left[x_{n}\right]
$$

where $\mathbf{a}_{n}=\operatorname{Cov}\left(x_{n}, \mathbf{z}_{n}\right) \operatorname{Cov}\left(\mathbf{z}_{n}, \mathbf{z}_{n}\right)^{-1}$. The expectations and covariances of symbols are computed from the input a priori LLRs $L_{a}^{E}$. The time varying MMSE solution consists thus of a soft interference canceller which cancels both the linear and non linear ISI.

The estimated symbols are assumed to be function of the transmitted symbols as $\hat{x}_{n}=\kappa_{n} x_{n}+e_{n}$ where $\kappa_{n}=\operatorname{Cov}\left(\hat{x}_{n}, x_{n}\right)$ and $e_{n}$ is a Gaussian noise with $\operatorname{var}\left(e_{n}\right)=\kappa_{n}^{*}-\left|\kappa_{n}\right|^{2}$.

The time varying MMSE solution raises two complexity issues. On the one hand, the matricial inversion grows as $O\left(N^{3}\right)$ which is a common complexity limitation for linear channels as well. On the other hand, computing the covariances of third order Volterra symbols requires heavy computation of expectations of products of four to six symbols for each estimated symbol. Thus, in order to reduce the complexity of the exact MMSE solution, we investigated time invariant MMSE solutions which will be detailed hereafter.

1) Suboptimal No-Apriori (NA) MMSE: The first reduced complexity time invariant MMSE solution is computed using the assumption that no-apriori information is available in the equalizer which results in the following expression:

$$
\mathbf{a}_{N A}=\mathbf{h}_{n}^{H}\left(\mathbf{H H}^{H}+\left(\sigma_{w}^{2}+\sigma_{\tilde{w}}^{2}\right) \mathbb{I}_{N}\right)^{-1}
$$

where $\mathbf{h}_{n}=\mathbf{H} \times \mathbf{u}_{n}$ and $\mathbf{u}_{n}=\left[\mathbf{0}_{1 \times N_{1}+M-1}, 1, \mathbf{0}_{1 \times N_{2}}\right]^{T}$ and $\sigma_{\tilde{w}}^{2}=\sum_{i, j, k}\left|h_{i, j, k}\right|^{2}$. The estimation error variance is simplified by assimilating the non linear interference to a Gaussian noise which yields:

$$
\begin{aligned}
\operatorname{var}\left(e_{n}\right) & =\mathbf{a}_{N A}\left(\mathbf{H} \operatorname{Cov}\left(\mathbf{x}_{n}, \mathbf{x}_{n}\right) \mathbf{H}^{H}+\left(\sigma_{w}^{2}+\sigma_{\tilde{w}}^{2}\right) \mathbb{I}_{N}\right) \mathbf{a}_{N A}^{H} \\
& -\left|\kappa_{n}\right|^{2}
\end{aligned}
$$

2) Low complexity (LC) MMSE: The idea is to compute the MMSE filter which minimises $\frac{1}{L} \sum_{n=0}^{L-1} E\left[\left|\hat{x}_{n}-x_{n}\right|^{2}\right]$. The optimal solution becomes:

$$
\mathbf{a}_{L C}=\left(\frac{1}{L} \sum_{n=0}^{L-1} \operatorname{Cov}\left(x_{n}, \mathbf{z}_{n}\right)\right)\left(\frac{1}{L} \sum_{n=0}^{L-1} \operatorname{Cov}\left(\mathbf{z}_{n}, \mathbf{z}_{n}\right)\right)^{-1}
$$

The structure of the LC equalizer can be efficiently implemented neglecting both the border effects and third order covariances yielding:

$\mathbf{a}_{L C}=\mathbf{u}_{n}^{T} \mathbf{H}^{H}\left(\bar{v} \mathbf{H} \mathbf{H}^{H}+(1-\bar{v}) \mathbf{h}_{n} \mathbf{h}_{n}^{H}+\left(\sigma_{w}^{2}+\sigma_{\tilde{w}}^{2}\right) \mathbb{I}_{N}\right)^{-1}$

where we define the mean variance as: $\bar{v}=\frac{1}{L} \sum_{n=0}^{L-1} \operatorname{var}\left(x_{n}\right)$. The estimation error variance is simplified as for the NoApriori approximation.

\section{Frequency Domain (FD)-MMSE}

In order to further reduce the complexity of the turbo equalizer for the non linear satellite channel, block-wise frequency domain processing has been investigated in [7]. To do so, a CP of length at least equal to channel length $(M-1)$ is appended to each block of $L$ transmitted symbols. At the receiver, after removing the $\mathrm{CP}$, each block of symbols is transformed to the frequency domain by a Fast Fourier Transform (FFT) of size $L$. A frequency domain representation of the non linear channel has been presented in [10] as follows:

$$
\begin{aligned}
Z_{m}= & H_{m} X_{m}+\sqrt{L} \sum_{p=0}^{L-1} \sum_{q=0}^{L-1} \sum_{r=0}^{L-1} H_{p, q, r}^{(3)} X_{p} X_{q} \bar{X}_{r} \\
& \delta_{N}(p+q+r-m)
\end{aligned}
$$


where $Z_{m}, H_{m}, X_{m}, \bar{X}_{m}$ are the $m$-th outputs of the $\mathrm{N}$ FFT of $\left[z_{0}, \ldots, z_{L-1}\right],\left[h_{0}, \ldots, h_{M-1}\right]$ and $\left[x_{0}, \ldots, x_{L-1}\right]$ $\left[x_{0}^{*}, \ldots, x_{L-1}^{*}\right]$ respectively, and $H_{p, q, r}^{(3)}$ is the $(p, q, r)$-th three dimensional L-FFT (3D-L-FFT) of the kernels $\left[h_{i, j, k}\right]$ and where the delta-function modulo $L$ is defined as $\delta_{L}(m)=$ 1 if $m=0$ modulo $[L]$ (the reader is referred to [7] for more detailed derivations).

The FD-MMSE estimated symbols write as follows:

$$
\begin{aligned}
\hat{X}_{m} & =\frac{H_{m}^{*}}{\sigma_{\tilde{w}}^{2}+\sigma_{w}^{2}+\left|H_{m}\right|^{2}} Z_{m} \\
& +\left(C-\frac{\left|H_{m}\right|^{2}}{\sigma_{\tilde{w}}^{2}+\sigma_{w}^{2}+\left|H_{m}\right|^{2}}\right) E\left[X_{m}\right] \\
& -\frac{H_{m}^{*}}{\sigma_{\tilde{w}}^{2}+\sigma_{w}^{2}+\left|H_{m}\right|^{2}} \sum_{p=0}^{L-1} \sum_{q=0}^{L-1} \sum_{r=0}^{L-1} H_{p, q, r}^{(3)} E\left[X_{p} X_{q} \bar{X}_{r}\right] \\
& \sqrt{L} \delta_{L}(p+q+r-m)
\end{aligned}
$$

where $C=\frac{1}{N} \sum_{m=0}^{L-1} \frac{\left|H_{m}\right|^{2}}{\sigma_{w}^{2}+\sigma_{\tilde{w}}^{2}+\left|H_{m}\right|^{2}}$.

The frequency domain MMSE solution consists thus of a scalar multiplication of the input frequency domain symbols, treating only the linear part, whereas the non linear part is considered as additive noise with variance $\sigma_{\tilde{w}}^{2}$. It should be noted however, that the application of a frequency domain equalizer relies on the assumption that a $\mathrm{CP}$ has been appended to the transmitted blocks with a subsequent spectral efficiency loss characterised by the ration $1+C P / L$. For large block size $L$ and small channel memory size $M$, this loss is negligible. It is thus interesting to question the sensitivity of the frequency domain equalizers towards a $\mathrm{CP}$ mismatch i.e. when the $\mathrm{CP}$ duration is shorter than the channel memory or even null which makes time and frequency domain solutions more comparable in terms of transmitter architecture and spectral efficiency.

\section{Volterra soft demapper}

One of the least complex receivers for the Volterra channel consists of a soft demapper considering both the linear and non linear interference as Gaussian noise leading to an expression of the probability of symbols:

$$
P\left(x_{n}=\tilde{x} \mid \mathbf{z}\right) \propto \exp \left(\frac{\left|z_{n}-h_{0} \tilde{x}\right|^{2}}{\sigma_{w}^{2}+\sigma_{v}^{2}}\right)
$$

where $\sigma_{v}^{2}=\sum_{i=1}^{M-1}\left|h_{i}\right|^{2}+\sigma_{\tilde{w}}^{2}$ is the overall Volterra interference power which is assumed uncorrelated of the symbol $x_{n}$. This assumption is of course far from being realistic but yields simplified equalizer processing.

\section{E. Complexity comparison}

Unlike iterative receivers for a linear channel, the complexity of the non linear Volterra channel equalizers does not only depend on the channel memory but on the number of non linear kernels as well. The respective complexities of these equalizers are provided hereafter for a block of $L$ estimated symbols taking only into consideration the complexity of the equalizer coefficients and the computation of estimates:
TABLE I

VOLTERRA KERNELS FOR THE TEST CHANNEL

\begin{tabular}{|l|l|}
\hline $1 s t$ order kernels & $3 r d$ order kernels \\
\hline$h_{0}=-0.0802+0.9387 i$ & $h_{0,0,1}^{(3)}=-0.0091-0.0232 i$ \\
$h_{1}=-0.0108+0.1652 i$ & $h_{0,0,3}^{(3)}=-0.0030-0.0108 i$ \\
$h_{2}=0.0165-0.1500 i$ & $h_{1,1,0}^{(3)}=-0.0168-0.0453 i$ \\
$h_{3}=-0.0126+0.1398 i$ & $h_{1,1,2}^{(3)}=0.0077+0.0271 i$ \\
& $h_{2,2,1}^{(3)}=0.0045+0.0087 i$ \\
& $h_{2,2,0}^{(3)}=-0.0031-0.0088 i$ \\
& $h_{2,2,1}^{(3)}=0.0045+0.0087 i$ \\
\hline
\end{tabular}

- MAP equalizer $\approx O\left(L M^{\mathcal{M}}\right)$

- Exact MMSE solution $\approx O\left(L N^{3}\right)+O\left(N^{2} L\left|\mathcal{I}_{3}\right|\right)$

- NA-MMSE solution $\approx O(L N)$

- FD-MMSE solution $\approx O\left(L \log _{2}(L)\right)$

- Volterra soft demapper $\approx O(L)$

It can be noted that the FD equalizer is beneficial over the NAMMSE for long memory channels requiring longer filter size $N$. Some numerical applications will be given in the following section.

\section{Simulation RESUlts}

In order to compare the performance and complexity of these equalizers let us consider the following system specifications. We consider a rate $1 / 2$ Low Density Parity Check (LDPC) code with the short codeword length 16200. The mapper consists of the DVB-S2 16-APSK modulation as presented in [11]. A root raised cosine transmit filter of 0.2 roll-off is used and an Input Back-Off equal to $I B O=1.2 \mathrm{~dB}$ is assumed. The HPA model relies on Saleh's amplifier model using $\alpha_{a}=1.9638, \beta_{a}=0.9945, \alpha_{\phi}=2.5293$ and $\beta_{\phi}=2.8168$. The equivalent Volterra kernels are obtained from the procedure explained in Sec. II and are shown in Table I.

Given the filter length $N=7$, the complexity of the FDMMSE is smaller or equivalent to that of the time domain equalizers as long as $L \leq 256$. The $\mathrm{CP}$ length is equal to channel memory $M-1=3$. In the sequel, two FFT sizes are investigated $L=16$ yielding a spectral efficiency loss of $0.75 \mathrm{~dB}$ and $L=256$ with a loss of $0.05 \mathrm{~dB}$. Given these numerical values, the complexity of the MAP equalizer is approximately $1.10^{6}$, the exact MMSE $8.10^{4}$ and $2.10^{3}$ for both the NA-MMSE and FD-MMSE for $L=256$. The notation (A,B) designates $A$ equalization iterations and $B$ LDPC decoder inner iterations. Figure 4 plots the Bit Error Rate (BER) performance of the selected satellite test channel for the first turbo-iteration i.e. $(1,50)$. On the one hand, there is a $1 \mathrm{~dB}$ gap between the Volterra soft demapper compared to the least performing MMSE equalizer. On the other hand, the first iteration of the FD-MMSE equalizer slightly outperforms that of the equivalent time domain implementation for $L=256$ although being equivalently complex. This stems from the fact that a block of $L$ symbols is exploited to equalize one symbol in the FD-MMSE, when only $N<L$ symbols are used to 


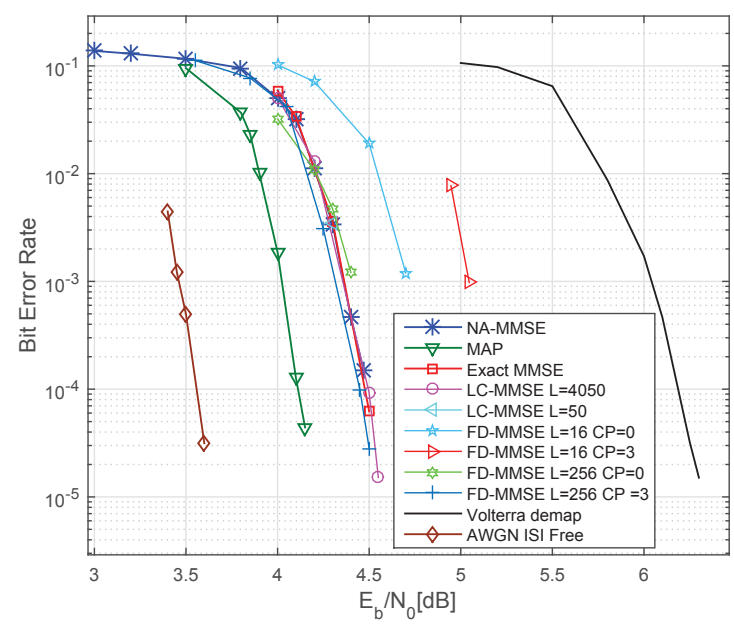

Fig. 3. Comparison of the non iterative receivers $(1,50)$

generate one time domain estimated symbol. However, the FDMMSE with $L=16$ has poorer performance compared to the NA-MMSE because of the spectral efficiency loss which is taken into account in the ratio $E_{b} / N_{0}$. A CP mismatch for $L=16$, enhances the performance due to the efficiency gain, whereas it slightly degrades the performance for large FFT sizes $L=256$. Ideally, an optimal CP size should be found in order to allow for the best efficiency-performance gain. The LC-MMSE performance is similar for different values of the parameter $L=50$ and a full block length $L=4050$. Figure 4 depicts the performance of the iterative receivers for the scheduling $(50,1)$. It can be noted that the gap between FDMMSE and NA-MMSE is reduced since the size of the feed forward length is no longer a limiting factor thanks to the soft ISI cancellation. Furthermore CP omission does not degrade much the performance of the FD-MMSE $L=256$. As for the LC-MMSE, it is shown that the smaller the block size the slightly better the performance are. Globally, linear equalizers provide interesting performance which are only degraded by $0.3 d B$ compared with the optimal equalizer while being $9.10^{3}$ times less complex than the optimal equalizers.

\section{CONCLUSION}

This article presented a comparison of different iterative receivers for the non linear satellite channel. Three indicators have been taken into account: complexity, spectral efficiency and performance. Among these receivers, the frequency domain implementation offers good performance-complexity trade-off for long memory channels. However, frequency domain equalizers are subject to an efficiency loss due to the addition of the CP. The omission of the $\mathrm{CP}$ enhances the performance of small block sizes FD processing while slightly degrading that of large block sizes FD equalizers. Thus, an optimal (FFT size, CP size) operating point allowing a good complexity, efficiency and performance trade-off needs to be searched for for a given channel configuration.

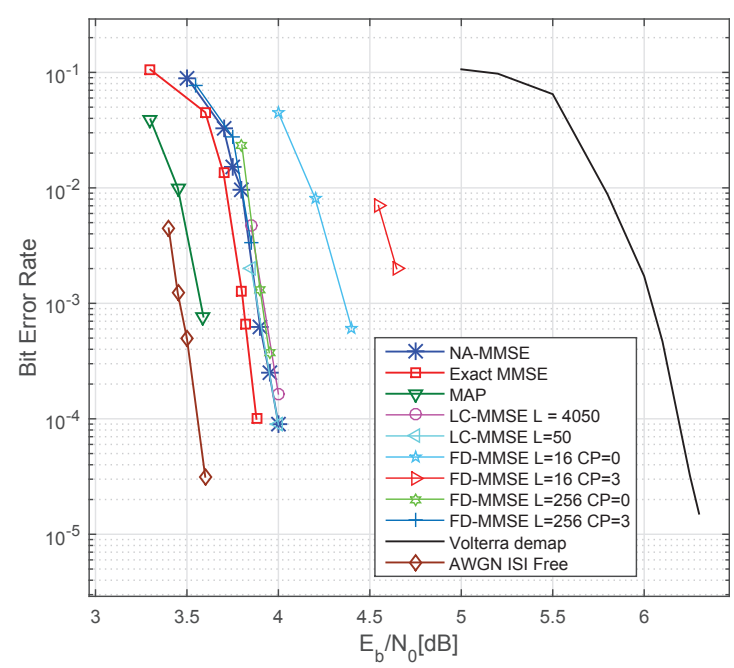

Fig. 4. Comparison of the iterative receivers performance $(50,1)$

\section{REFERENCES}

[1] 302 307-2 ETSI EN, "Digital Video Broadcasting (DVB), DVB Document A83-2, Second generation framing structure. channel coding and modulation systems for Broadcasting. Interactive Services. News Gathering and other Broadband satellite application, Part II: S2-Extensions (DVB-S2X)," European Standard (Telecommunications series), 032014.

[2] S. Benedetto, E. Biglieri, and R. Daffara, "Modeling and performance evaluation of nonlinear satellite links-a volterra series approach," IEEE Transactions on Aerospace and Electronic Systems, vol. AES-15, no. 4, pp. $494-507$, july 1979

[3] Y.T. Su, Mu-Chung Chiu, and Yen-Chih Chen, "Turbo equalization of nonlinear TDMA satellite signals," in Global Telecommunications Conference, 2002. GLOBECOM 'O2. IEEE, nov. 2002, vol. 3, pp. 2860 - 2864 vol.3.

[4] A. Gutierrez and W.E. Ryan, "Performance of volterra and MLSD receivers for nonlinear band-limited satellite systems," IEEE Transactions on Communications, vol. 48, no. 7, pp. $1171-1177$, jul 2000.

[5] G. Colavolpe and A. Piemontese, "Novel SISO detection algorithms for nonlinear satellite channels," IEEE Wireless Communications Letters, vol. 1 , no. 1 , pp. $22-25$, february 2012.

[6] B. Benammar, N. Thomas, C. Poulliat, ML. Boucheret, and M. Dervin, "On linear MMSE based turbo-equalization of nonlinear volterra channels," in 2013 IEEE International Conference on Acoustics, Speech and Signal Processing (ICASSP),, 2013, pp. 4703-4707.

[7] B. Benammar, N. Thomas, C. Poulliat, M.-L. Boucheret, and M. Dervin, "Asymptotic Analysis and Design of Iterative Receivers for Non Linear ISI Channels," in The 8th International Symposium on Turbo Codes \& Iterative Information Processing, August 2014.

[8] C. Douillard, M. Jezequel, and C. Berrou, "Iterative correction of intersymbol interference: Turbo equalization," European Transactions on Telecommunications, vol. 6, no. 5, pp. 507 a 511, Sept. Oct 1995.

[9] L. Bahl, J. Cocke, F. Jelinek, and J. Raviv, "Optimal decoding of linear codes for minimizing symbol error rate (corresp.)," IEEE Transactions on Information Theory, vol. 20, no. 2, pp. 284-287, Mar 1974.

[10] Sungbin Im, "Adaptive equalization of nonlinear digital satellite channels using a frequency-domain volterra filter," in Military Communications Conference, 1996. MILCOM '96, Conference Proceedings, IEEE, Oct 1996, vol. 3, pp. 843-848 vol.3.

[11] V1.2.1 (2009-08) 302307 ETSI EN, "Digital video broadcasting (DVB); second generation framing structure, channel coding and modulation systems for broadcasting, interactive services, news gathering and other broadband satellite applications (DVB-S2)," European Standard (Telecommunications series), 082009. 